\title{
Polyneuritis cranialis: clinical and electrophysiological findings
}

\author{
Alberto Polo, Paolo Manganotti, Giampietro Zanette, Domenico De Grandis
}

\begin{abstract}
A 13 year old boy, developed bilateral facial weakness, dysphonia and dysphagia acutely after a febrile illness. Neurological examination and MRI of the brain were normal. The CSF protein level increased. Blink reflex monitoring during clinical recovery was consistent with demyelination of the lower cranial nerves innervating the branchial arch musculature, a rare variant of Guillain-Barré syndrome.
\end{abstract}

Guillain-Barré syndrome has been subdivided into several neurological syndromes. Cranial muscles may be variably affected later in the course of the disease. Sometimes weakness of cranial muscles is observed at the onset of the clinical picture, legs and arms being affected little, if at all, during development of the illness. Acute ophthalmoplegia is usually associated with ataxia and generalised areflexia in the Miller-Fisher variant. More widespread cranial nerve involvement with facial weakness, dysarthria and dysphagia may be present in the full syndrome. This variable clinical course emerges from a review of more than 100 cases of Miller-Fisher syndrome in the literature. ${ }^{10}$ An unusual clinical variant characterised by pharyngeal-cervical-brachial weakness is also described by Ropper. ${ }^{8}$ Clinical findings confined to involvement of lower cranial nerves are very rare. We report a course of "polyneuritis cranialis" whose electrophysiological and MRI findings suggested a demyelinating disorder affecting the lower cranial nerves.

\section{Patient report}

A 13 year old boy with no history of neurological or infectious disease presented with a period of generalised weariness. After a short non-specific rise in temperature which settled spontaneously without any other systemic symptoms, he experienced unpleasant sensations affecting the tongue and oral cavity, lasting for two days. At the same time his speech became slurred and he began to complain of difficulty in swallowing. On day 6 after onset the CSF (albumin $200 \mathrm{mg} / 1,1 \mathrm{cell} / \mu \mathrm{l}$ ) and CT scan were normal. On admission on day 8 he was alert and orientated. He showed a symmetrical facial diplegia with Bell's phenomenon, marked impairment of mastication and deglutition, aphonia, palatal palsy, reduced lateral movements of the tongue and inability to protrude the tongue beyond the dental arch. Sternocleidomastoid and trapezius muscle strength were preserved. Individual eye movements and both pupil reaction to light and accommodation were normal. His gait was slightly unsteady. The tendon reflexes were present in all four limbs. Cutaneous reflexes were normal. Joint position sense, pinprick and light touch sensation were normal, and no sensation disorders were detectable in territories depending on the fifth and lower cranial nerves.

The general examination was unremarkable. The patient's height was $160 \mathrm{~cm}$ and his weight was $48 \mathrm{~kg}$; blood pressure values were $120 / 80 \mathrm{~mm} \mathrm{Hg}$. He showed no tachycardia, postural hypotension, or any other autonomic dysfunction. Sphincter function was normal, as were conventional blood chemistry parameters. Chest and skull $x$ rays and EEG were also normal.

At day 10 his swallowing difficulty had worsened. The patient could be fed only by nasogastric tube: He showed bilateral facial plegia, as well as marked masseter and temporal hypotrophy associated with bulbar palsy. NMR revealed no pathological signs. A second lumbar puncture was performed and revealed only a protein increase $(730 \mathrm{mg} / \mathrm{l}$ protein, no cells). On day 22 initial signs of recovery were observed: although phonation was still compromised, he was able to articulate a few sentences, swallow a few teaspoonfuls of liquid and move the lips slightly. He was able to close his eyes slowly but completely. The deep tendon reflexes persisted normally. Four days later the nasogastric tube was removed and normal feeding was resumed. $\mathrm{He}$ weighed $42.5 \mathrm{~kg}$.

Thirty days after onset of symptoms the patient showed a further improvement: speech was comprehensible despite persistence of dysphonia and he was able to puff out his cheeks. Mastication movements and tongue protrusion beyond the dental arch were possible and the deglutition difficulty had decreased. At discharge on day 40 the neurological examination was normal outside the lower cranial nerves. At this level there was masseter and temporalis muscle hypotrophy with decreased masticatory strength. The facial weakness was mildly asymmetrical. The pharyngeal reflex was torpid. Dysphonia and mild dysphagia persisted. Although atrophy of the tongue was present, lateral tongue movements and protrusion were possible. Five months 


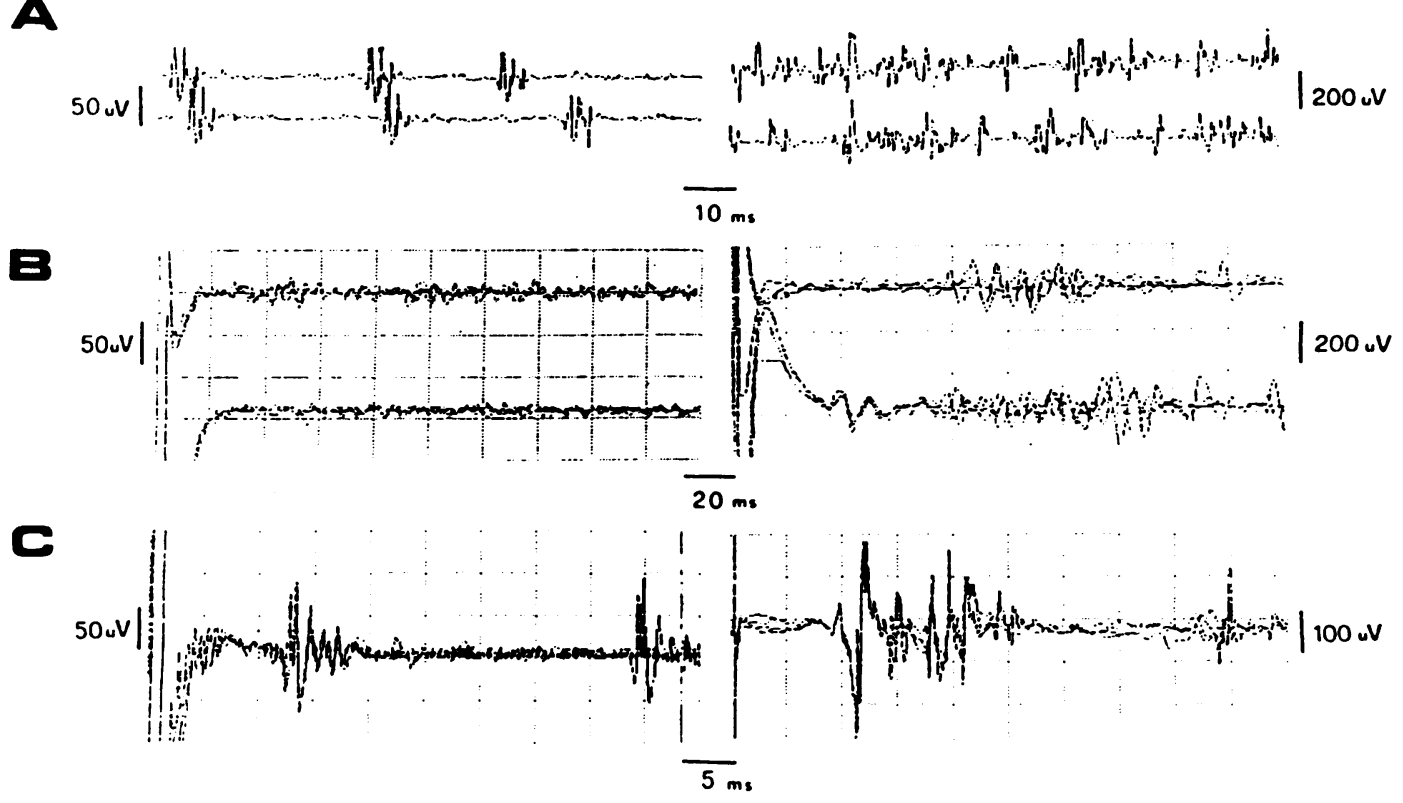

Figure 1 On admission A) Single unit interference pattern of orbicularis oculi; B) Blink reflex: R1 and R2 components are absent; C) MAP from the orbicularis oculi delayed in latency and reduced in amplitude. 2) Five months later. A) Improvement in interference and in motor unit; B) Blink reflex elicitable; C) Recovery of both latency and amplitude of the $M A P$.

later the patient showed distinct clinical improvement. The gag reflex was present, and there were no abnormalities in deglutition, mastication or tongue movements. The masseter muscle was palpable and presented good tonus. Complete eye closure was possible; the patient could whistle and smile.

\section{Electrophysiological studies}

On admission, needle electromyography (EMG) showed no evidence of spontaneous activity of facial muscles, and a single unit interference pattern appeared during maximal voluntary contraction of the orbicularis oculi (fig 1A). Blink reflex was elicited ${ }^{5}$ and both $\mathrm{Rl}$ and R2 components were absent (fig 1B). On stimulating the facial nerve at the tragus, the MAP (motor action potential) from the orbicularis oculi was delayed in latency $(11.80 \mathrm{~ms})$ and reduced in amplitude (100 uV) (fig 1C). Both the latency and amplitude of the MAPs recorded from the trapezius and sternocleidomastoid muscles were normal. Conventional nerve conduction and EMG parameters were normal in territories depending on the spinal nerves. Late responses demonstrated high persistence and normal latency of $F$ waves in response to both median and peroneal nerve stimulation. SEPs from the median and tibialis posterior nerve and BAEPs showed symmetrical responses with a normal central conduction time. Five months later, the recruitment pattern from the orbicularis oculi showed an improvement in interference and in motor unit amplitude (fig 2A); blink reflex could be elicited (fig 2B); the $R \mathbf{l}$ latency was significantly increased (17 ms, normal upper limits $13 \mathrm{~ms}$ ). Partial recovery of both latency $(8.4 \mathrm{~ms})$ and amplitude $(400 \mathrm{uV})$ of the MAPs from the orbicularis oculi (fig 2C) was observed.

\section{Discussion}

Both the Miller-Fisher syndrome and polyneuritis cranialis are thought to be variants of acute idiopathic polyneuritis. ${ }^{2}$ The MillerFisher syndrome is still subject to debate in an attempt to explain the relationship between the clinical manifestations and the site of the lesion. $^{17}$ In the opinion of most authors, however, little doubt exists that all the manifestations of Miller-Fisher syndrome can be explained by involvement of both spinal and cranial nerves, without any brainstem lesion necessarily being involved. ${ }^{3910}$ Polyneuritis cranialis is occasionally encountered in the literature, especially in its pure clinical forms. Van Bogaert and Moore ${ }^{12}$ described three cases of GBS confined to the cranial nerves, while another four patients were observed by Munsat and Barnes. ${ }^{6}$ The presence of additional symptoms, such as ataxia and areflexia, suggests a transitional form of acute polyneuritis. ${ }^{511}$ The case illustrated in our paper was characterised by bilateral, almost symmetrical, lower cranial nerve palsy. The clinical course, the CSF albumino-cytological dissociation and the blink reflex monitoring suggested primary demyelinating disease affecting these cranial nerves. CNS involvement was excluded because by the absence of relevant clinical features, normal MRI scan and normal evoked potentials. The spinal peripheral nerves were spared. 
The patient may have been affected by a cranial nerve variant of Guillain-Barré syndrome. The neurological features were strictly confined to muscles innervated by the 5 th, 7 th, 9th, 10th and 12th pairs of cranial nerves. Interestingly, all these nerves, except for the 12th pair, innervate muscles of specific embryological origin, that is, the branchial muscles; conversely, the 12th pair innervates muscles included in the somitic group, which are functionally similar to spinal-nervedependent muscles. From the functional, as opposed to the anatomical point of view, our patient may represent a typical case of polyneuritis cranialis as proposed by Asbury. ${ }^{2}$

1 Aldin AN, Anderson M, Bickerstaff ER, Harvey I. Brainstem encephalitis and the syndrome of Miller Fisher. A stem encephalitis and the syndrome of
clinical study. Brain 1982;105:481-95.

2 Asbury AK. Diagnostic consideration in Guillain-Barré syndrome. Ann Neurol 1981;9:1-5.
3 Juncos JL, Beal MF. Idiopathic cranial polyneuropathy: a fifteen year experience. Brain 1987;110:197-211.

4 Kimura J. The blink relex. In: Kimura J, ed. The electrodiagnosis in disease of nerve and muscle: principles and practice. Philadelphia: Davis FA, 1985:323-51.

5 McFarland HR. Polyneuritis cranialis as the sole manifestation of Guillain-Barré syndrome. Mo Med 1976;73: 227-9.

6 Munsat TL, Barnes JE. Relation of multiple cranial nerve dysfunction to the Guillain-Barré syndrome. $f$ Neurol Neurosurg Psychiatry 1965;28:115-20.

7 Pessin MS, Logigian CL, Brown MT, Shuren JE, Kelly JJ, Barbas NR. CNS dysfunction in Fisher's syndrome. Neurology 1989;39:998-9.

8 Ropper AH. Unusual clinical variants and signs in GuillainBarré Syndrome. Arch Neurol 1986;43:1150-2.

9 Ropper AH. Three patients with Fisher's syndrome and normal MRI. Neurology 1988;38:1630-1.

10 Sauron B, Bouché P, Cathala HP, Chain F, Castaigné P. Miller Fisher syndrome: Clinical and electrophysiologic evidence of peripheral origin in 10 cases. Neurology 1984;34:953-6.

11 Shuaib A, Beker WJ. Variants of Guillain-Barré syndrome: Miller Fisher syndrome, facial diplegia and multiple

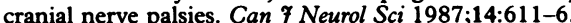

12 Van Bogaert L, Maere $M$. Les polyradiculonevrites craninnes bilaterales avec dissociation albumino-cytologique: forms craniennes des polyradiculonevrites du type que: forms craniennes des polyradiculonevrites du type 275-81. 